\title{
La consulta ginecológica en la adolescencia bajo la óptica de médicos ginecólogos de la ciudad de Río de Janeiro
}

\author{
Sandra de Morais Pereira, $P h D^{1}$, Stella R. Taquette, $P h D^{2}$ \\ 1 Servicio de Ginecologia Infanto-Puberal, Hospital Federal de Bonsucesso (Ministério da Saúde/Brasil). Universidad del \\ Estado de Rio de Janeiro, Brasil. ${ }^{2}$ Universidad de São Paulo, Ribeirão Preto, Facultad de Ciencias Médicas, Universidad \\ del Estado de Rio de Janeiro, Brasil.
}

\section{RESUMEN}

Objetivo: Conocer actitudes y conductas de los ginecólogos en la atención de adolescentes y evaluar las dificultades/facilidades que encuentran. Metodología: Estudio de corte transversal realizado mediante un cuestionario estructurado, con muestra significativa de médicos asociados a la Associação de Ginecologia e Obstetrícia do Estado do Rio de Janeiro. Los participantes respondieron preguntas sobre datos personales, ubicación del ejercicio de la profesión, procedimientos médicos, experiencias y actitudes en la consulta ginecológica y también brindaron su opinión sobre la necesidad de un entrenamiento específico para la atención de este grupo de usuarios. Se ha utilizado el test Chi-cuadrado para la comparación entre proporciones. Resultados: Hubo predominancia de los participantes del sexo femenino $(62,3 \%)$, edades entre los 25 y 35 años $(39,3 \%)$ y con más de 20 años de conclusión de curso académico $(38,2 \%)$. La contracepción fue la razón más frecuente de la consulta de las adolescentes. Se observó, cierto temor de algunos en cuanto a la indicación del método hormonal a esta población. El principal obstáculo a la atención de las adolescentes señalado por los entrevistados fue el mayor tiempo de duración de la consulta. Los participantes pusieron de relieve la importancia de la capacitación profesional específica hacia la atención de adolescentes y de la realización del examen colpocitológico rutinariamente. Conclusión: Los médicos ginecólogos estiman que la asistencia ginecológica de calidad para adolescente incluye entrenamiento profesional específico, pues la consulta ginecológica es un espacio privilegiado para la promoción de la salud y prevención de agravios derivados de la práctica sexual sin protección.

\section{PALABRAS CLAVE: Adolescencia, consulta, ginecología}

\section{SUMMARY}

Objectives: To know the attitudes and procedures of gynecologists who attend teenagers and to evaluate the difficulties/facilities found in the work with this sort of patients. Methodology: A study of transverse cut achieved through a structured list of questions, with prominent sample of doctors associated to the Gynecology and Obstetrics Association of Rio de Janeiro. The participants answered questions about personal data, working place, medical procedures, previous experience and location in gynecological attendance. They also gave their opinion about the necessity of specific treatment to teenagers. The Chi-square test was also utilized to make comparisons of proportions. Results: There was a predominance of female participants $(62,3 \%)$, ages between 25 to $35(39,3 \%)$, and with over 20 year graduates $(38,2 \%)$. Contraceptive methods were the biggest reason for gynecological consultation among adolescents. Younger and female 
gynecologists as well as the ones recently graduated adopted a less conservative posture during the consultation. The main obstacle to the attendance pointed by the doctors was the biggest duration time of the consultation. The participants highlighted the importance of specific professional training to the attendance of adolescents and the importance of more frequently colpocytologic exams. Conclusion: In gynecologists' point of view, a good gynecological care towards adolescents includes specific professional training, since the gynecological consultation is a privileged area to promote health and prevention of problems originated from unprotected sexual habits.

\section{KEY WORDS: Adolescence, consultation, gynecology}

\section{INTRODUCCIÓN}

La adolescencia se caracteriza como un periodo de transformaciones y se vuelve objeto sistemático de una serie de cuidados, atenciones e intervenciones, con el intento de garantizar una transición satisfactoria a la vida adulta que atienda a determinadas expectativas sociales (1). Aunque los programas de atención a este público ya están siendo puestos en funcionamiento hace mucho, se observan cambios significativos en el conjunto de rasgos peculiares que caracterizan la morbimortalidad de esta población, con un aumento de problemas que podrían evitarse por medidas de prevención y de promoción de salud.

Algunos profesionales presentan dificultad con las cuestiones propias de la clientela adolescente, que hacen cargo de la concientización y compromiso con la prevención de los agravios a la salud, y tienen como foco la iniciación sexual precoz, los riesgos de enfermedades de transmisión sexual y el embarazo no planeado (2).

En el intento de contribuir para la mejora de la atención prestada a esta población, las autoras buscaron estudiar la relación del médico con la adolescente durante la consulta ginecológica, con el objetivo de evaluar las principales dificultades existentes y conocer las actitudes y las conductas de los ginecólogos al atender a este público.

\section{MATERIAL Y MÉTODOS}

Se trata de un estudio de corte transversal hecho a través de un cuestionario estructurado. La investigación se desarrolló junto a una muestra representativa de ginecólogos comúnmente matriculados en la Asociación de Ginecología y Obstetricia del Estado de Río de Janeiro (SGORJ) y que tienen sus catastros actualizados, incluyendo dirección electrónica.

Los criterios de inclusión adoptados tuvieron el fin de obtener una muestra que fuera heterogénea $y$, por lo tanto, representativa. Fueron selecciona- dos aquellos que atienden en ginecología; sin restricción de edad; de ambos sexos; independiente de los años de experiencia profesional, que ejercen sus actividades en el sector público y/o privado y cuya clientela está compuesta por pacientes adultos y adolescentes. El incumplimiento de cualquiera de los criterios descritos se convirtió en el elemento de exclusión para este estudio.

En primer lugar se realizó un estudio piloto para el perfeccionamiento del instrumento. La recopilación de datos se llevó a cabo desde julio de 2009 a mayo de 2010. Los ginecólogos fueron invitados a participar a través de un mensaje por correo electrónico, de SGORJ, que contenía las explicaciones sobre el estudio, el cuestionario y también el término para dar su consentimiento. Se juzgó este recurso como más adecuado, en razón de la existencia de cuestiones posibles de coacción si fueran respondidas en presencia de un entrevistador.

El guión se dividió en dos partes y se elaboró a partir de los campos de conocimiento para el estudio. Primeramente se han incluido los datos personales como: edad, sexo, estudios, tiempo de ejercicio de la profesión y también la ubicación de la práctica profesional (servicio público y/o privado). En la segunda parte se abordaron los temas pertinentes a la práctica del entrevistado, además de la observación de sus conductas, experiencias y actitudes ante los diversos aspectos presentes en el contexto de la consulta ginecológica hacia esta población. En esta última fase, se cuestionaron los participantes cuanto a la relación médico y adolescente, según sus expectativas e impresiones. Se interrogaron sus opiniones en cuanto a la necesidad de un entrenamiento específico para la atención a este grupo de edad.

Para evaluar la confiabilidad del instrumento, se realizó el pre-test de las entrevistas en dos momentos distintos. Todas las variables estudiadas presentaron valores de porcentaje de conformidad por encima de los $90 \%$ de la estadística Kappa, que asegura, así, la confiabilidad y la validez del instrumento.

Este estudio fue previamente aprobado por el 
Comité de Ética en Investigación de la Universidad del Estado de Río de Janeiro (UERJ). Cumplió con los principios éticos que figuran en la Declaración de Helsinki y en la resolución 196/96 del Consejo Nacional de Salud.

\section{RESULTADOS}

Características de los ginecólogos entrevistados. Contestaron el estudio 191 ginecólogos. De estos, el $62,3 \%$ eran del sexo femenino. Los médicos más jóvenes ( $\leq 35$ años) representaron el $39 \%$ de los entrevistados; aquellos entre 36 y 59 años al $48,7 \%$ y los mayores de 60 años sumaron $12 \%$. Se señalaron algunas cuestiones propuestas con más de una opción, por eso hubo un número total $(\mathrm{N})$ de múltiples respuestas. La población del estudio fue evaluada en relación con el momento de su licenciatura y de éstos $61,2 \%$, tenían más de 11 años de licenciados. Este público, en su gran mayoría (65\%), tuvo la residencia médica como especialización. Algunos participantes $(29,8 \%)$ tenían otro diploma de postgrado, incluso $(20,4 \%)$ asistieron el postgrado Strictu Sensu (Maestría, Doctorado y Postdoctorado). Los participantes de la investigación contestaron que ejercían sus actividades tanto en situaciones públicas como privadas, siendo que la mayoría trabajaba en un consultorio privado $(60,4 \%)$.

La experiencia de los profesionales en la consulta ginecológica para la clientela adolescente. El principal motivo relatado por los entrevistados que requerían atención ginecológica en este grupo de edad, fue la búsqueda por orientación en anticoncepción $(48,9 \%)$, por infecciones ginecológicas $(30,8 \%)$, para acompañamiento prenatal $(12,2 \%)$ y por otros motivos $(8,1 \%)$, como alteraciones del flujo menstrual, exámenes de rutina y distintas dudas. Los médicos, sin embargo, consideran las enfermedades de transmisión sexual (ETS) $(46,8 \%)$ el tema más importante a abordar durante una consulta, luego la anticoncepción $(36,4 \%)$ y orientaciones sobre la higiene $(13,6 \%)$.

Los investigados afirmaron que la orientación anticoncepcional debería iniciarse luego de la menarquía $(47,1 \%)$ y que se debería enfatizar la importancia del uso de condones ya en la primera consulta $(51 \%)$. La gran mayoría $(94,1 \%)$ prefirió explicar siempre a lo largo de la atención sobre la doble protección a las adolescentes. La indicación de la anticoncepción de emergencia (AE) ocurre, con preferencia, por falla del método que ya se estaba utilizando en $60,6 \%$ de los casos. En atención a la solicitud del paciente, y en la ausencia de métodos anticonceptivos, $81 \%$ de los médicos prefieren prescribir anticonceptivos hormonales. En la
Tabla I, se puede verificar como fueron descritas las orientaciones anticoncepcionales.

En la práctica clínica de los ginecólogos, las ETS más encontradas en este grupo de edad fueron: VPH $83,8 \%$; clamidia $8,9 \%$ y sífilis $3,1 \%$. Sobre el examen colpocitológico, el $80,4 \%$ de los médicos respondieron que se lo debería realizar luego de que la joven inicia su actividad sexual. Los ginecólogos (43,0\%) consideraron importante la calidad de la consulta ginecológica para prevención del cáncer cervical.

Opinión de los entrevistados sobre la asistencia prestada a las adolescentes. Las opiniones de los ginecólogos sobre la atención a las adolescentes fueron diversas, como se observa en la Tabla II. Se consideró como principal traba el hecho de ser una consulta más prolongada $(36,2 \%)$. Y gran parte de los participantes $(58,8 \%)$ respondió que sería la baja incidencia de patologías graves la causa que facilita la atención a esta población.

Sobre la calidad de la consulta ginecológica, alrededor de la mitad de los médicos $(46,1 \%)$ respondió que sería importante un entrenamiento específico para aquellos profesionales que desean atender a este grupo de edad; mientras $21,5 \%$ de ellos piensan que sería mejor si se ofreciera una capacitación para todos con el fin de tener condiciones para la atención de adolescentes; $27,7 \%$ afirmaron ser suficiente sólo la motivación del médico y sólo $4,7 \%$ juzgaron innecesaria esta habilitación.

\section{DISCUSIÓN}

En las últimas décadas, ha habido un crecimiento de la investigación científica en las áreas de las ciencias humanas y biológicas. Se ha dado gran énfasis al estudio de la adolescencia, periodo en que la capacidad reproductiva y las habilidades cognitivas y sociales de los individuos son adquiridas (3), enseñando la relevancia de la temática entre los profesionales de salud.

Aunque la muestra estudiada ha sido muy heterogénea y pueda considerársela representativa de los médicos ginecólogos, es posible que haya ocurrido algún sesgo de selección, que en este caso, llevaría a una proporción sobrestimada de médicos de postgrado. Sin embargo, la presencia de participantes con más títulos académicos no comprometería los resultados clínicos obtenidos, al contrario, sólo reforzaría los resultados significativos que hay en esta investigación. Se cree que las limitaciones del estudio fueron compensadas por el hecho de que el cuestionario fue respondido sin la presencia de los entrevistados, lo que brindó a los profesionales menor coacción a lo largo del llenado, volviendo las respuestas más confiables (4). 
Tabla I

DISTRIBUCIÓN DE LAS RESPUESTAS DE LOS MÉDICOS ENTREVISTADOS SOBRE LA ORIENTACIÓN EN ANTICONCEPCIÓN DURANTE LA CONSULTA GINECOLÓGICA DE LA ADOLESCENTE

\begin{tabular}{llcc}
\hline Variable & Categoría & Frecuencia & (\%) \\
\hline \multirow{4}{*}{ Anticoncepción } & Después de la menarca & 90 & 47,1 \\
& Después de la sexarca & 41 & 21,5 \\
& Evita en $<15$ años & 20 & 10,5 \\
& Cuando se solicita & 40 & 20,9 \\
Uso de condones (1) & Primera consulta & 106 & 51,5 \\
& Cuando se solicita & 47 & 22,8 \\
& Después del inicio de la actividad sexual & 33 & 16,0 \\
Doble protección (2) & Ante las dudas & 20 & 9,7 \\
& No orienta & 2 & 1,1 \\
& Siempre orienta & 176 & 94,1 \\
Píldora del día siguiente (3) & Cuando se solicita & 1 & 4,3 \\
& Algunos casos & 15 & 0,5 \\
& Después de la violación & 117 & 7,8 \\
& Falla en el método & 58 & 30,6 \\
& En la ausencia de anticoncepción & 3 & 1,6 \\
Anticoncepción hormonal (4) & No recomienda & 158 & 81,0 \\
& Cuando se solicita & 3 & 1,5 \\
& Acompañada por el responsable & 33 & 16,9
\end{tabular}

(1) $\mathrm{N}=206$ respuestas. (2) $\mathrm{N}=187$ respuestas. (3) $\mathrm{N}=193$ respuestas. (4) $\mathrm{N}=195$ respuestas.

Tabla II

\section{OPINIÓN DE LOS MÉDICOS ENTREVISTADOS SOBRE LA CONSULTA} GINECOLÓGICA EN LA ADOLESCENCIA

\begin{tabular}{llcc}
\hline Variable & Categoría & Frecuencia & $(\%)$ \\
\hline & Más remiso & 68 & 36,2 \\
Trabas (1) & Difícil relación & 13 & 6,9 \\
& Anticonceptivos para pacientes muy jóvenes & 27 & 14,4 \\
& Otros* & 46 & 24,4 \\
& Ninguno & 34 & 18,1 \\
Facilidades (2) & No hay & 40 & 20,6 \\
& Consulta rápida & 15 & 7,7 \\
& Ausencia de patologías graves & 114 & 58,8 \\
& Otros & 25 & 12,9 \\
\hline
\end{tabular}

(1) N = 188; 3 médicos no respondieron a esta pregunta. (2) N = 194; 3 entrevistados respondieron más de una opción. *Afirmaron considerar obstáculos a la asistencia de este grupo de edad: la presencia de madres dominadoras, la inmadurez de las jóvenes y la no adhesión al tratamiento propuesto. ${ }^{* \star}$ Opinaron que existe facilidad en esta atención debido a factores distintos como la aceptación de orientación educativa, fácil comunicación y relación. 
La predominancia de ginecólogos del sexo femenino de distintos grupos de edad, en este estudio, acompaña una tendencia ya verificada por otros trabajos. Machado (1997) (5), observó la existencia de una "transición de género" en la actividad médica en Brasil, ya que a partir de la década de los 70 ha ocurrido una elevada feminización en el área, además de una expansión en número, y se espera un crecimiento de profesionales mayores, como ha ocurrido en la población de un modo general. Trabajos sobre el perfil del médico brasileño comprobaron que la constitución etaria era predominantemente joven, con $65 \%$ de ellos menores de 45 años de edad y que ejercían sus actividades hace menos de 15 años $(5,6)$.

En lo relacionado a las actividades profesionales, gran parte demostró elevada experiencia, con especialización en el área, y ya habiendo cursado residencia considerada el patrón de oro dentro de la sociedad médica. Muchos de ellos, incluso, con otros cursos. Se observó también la sobrecarga de trabajo, constatada por el hecho de que parte ponderable del universo evaluado presentaba varios vínculos para su supervivencia. La consecuencia directa de eso es un gran desgaste físico y mental, que puede comprometer la calidad de atención. Estudios muestran que cada vez más este profesional pasó a vivir del trabajo asalariado o de los convenios con los planes privados de salud complementaria. Su sueldo alcanzó niveles muy bajos, y lo llevó a la acumulación de dos, tres o más empleos, lo cual demanda gran número de horas semanales de servicio $(5,7)$.

En relación a la orientación anticonceptiva dada por los ginecólogos con predominancia para las adolescentes ya sexualmente iniciadas, cabe resaltar que la madurez sexual de las jóvenes está ocurriendo precozmente (8), con conocimientos todavía inadecuados (9). Por esta razón, la orientación dada por el médico a aquéllas que ya tienen actividad sexual o están a punto de iniciarla, debe enfatizar las responsabilidades provenientes de esta decisión y también la prevención de las enfermedades trasmitidas sexualmente.

Se observó que la doble protección fue la orientación de casi todos los entrevistados. El uso de dos métodos es un procedimiento reciente y no aceptado por todas las usuarias. Por lo tanto compete a los ginecólogos evaluar las necesidades individuales y ayudar a la paciente para una decisión futura.

Aunque la mayoría de los participantes hubiera recetado anticonceptivos hormonales cuando lo pedía la joven, se observó, todavía, cierto temor de algunos en cuanto a la indicación del método hormonal a esta población. Sin embargo, se debe resaltar que el médico en la consulta y su paciente pueden decidir por la anticoncepción si la juzgan la mejor opción para la salud de la joven. Si sucede la prescripción de los anticonceptivos hormonales para las adolescentes menores de 14 años, se deberá tomar en cuenta la solicitud de la propia niña, respeto a los casos de elección, independiente de la edad, sin constituirse en acto médico ilícito (10).

Todavía hay un tabú en lo que se dice respecto a la prescripción de la anticoncepción de emergencia. Se creó esta forma de anticoncepción para usarse una sola vez, como una opción ante una situación de emergencia y no de forma regular en el control de natalidad, pues es menos eficaz que otras formas de anticoncepción. Aunque un número elocuente de investigadores afirmara prescribir este método siempre que hubiera una relación desprotegida, algunos entrevistados pusieron de relieve que sólo indicarían el método en situaciones de violación y una pequeña minoría no recomendaría jamás su empleo. Algunos ginecólogos argumentaron que al prestar información sobre este método a sus pacientes, permitirían que las jóvenes lo usaran de forma indiscriminada o incluso que cambiaran el uso del condón por esta opción de anticoncepción. Estudios sobre el tema afirman que la AE es usada por las jóvenes dentro de la prescripción y no hay cualquier indicador o tendencia al uso abusivo o indiscriminado de este método $(11,12)$.

En la experiencia clínica de los médicos, el virus del Papiloma Humano (VPH) se presentó como la más frecuente enfermedad transmitida sexualmente en la población adolescente, aunque hacen mención a la clamidia y a la sífilis, pero con menor ocurrencia. Datos de la Organización Mundial de la Salud (OMS) (13) relatan el aumento de la frecuencia de las ETS en edades cada vez más tempranas. Aproximadamente 40 tipos infectan la región genitoanal y otras mucosas corporales, siendo que algunos tipos se consideran de alto riesgo oncogénico $(14,15)$. En Estados Unidos es la enfermedad de transmisión sexual más frecuente, con alta prevalencia entre las jóvenes que ya empezaron su vida sexual (14). Muchas veces son infecciones asintomáticas y transitorias y pueden asociarse con anormalidades citológicas. Por lo general, el $90 \%$ de las infecciones recientes desaparecen en hasta 2 años (16). Sin embargo, persisten aquéllas con tipos de VPH de alto riesgo y que pueden progresar hacia lesiones precancerosas y en algunas ocasiones hasta el cáncer. Causa preocupación la creciente tasa de esta infección en este grupo de edad, ya que las jóvenes tienen muy poca o ninguna información. La población femenina todavía no sabe de las potenciales complicaciones a largo 
plazo causadas por esta infección, como el cáncer cervical (17).

La reciente aprobación de la vacuna para la prevención de la infección por este virus promete la reducción de ese tipo de cáncer (18). Persisten las controversias en relación al costo-beneficio, tiempo de protección y la necesidad de dosis de refuerzo (19). El American College of Obstetricians and Gynecologists (20) recomienda que la vacunación contra el VPH debe ofrecerse desde los 9 hasta los 26 años, pero enfatiza que debe mantenerse el rastreo citológico de forma regular.

En este público, los exámenes colpocitológicos suelen presentar más anormalidades en comparación con las mujeres adultas, pero con frecuencia son lesiones de menor gravedad y con altas tasas de regresión (21). Delante de este cuadro se percibe la importancia de que el examen colpocitológico sea ofrecido siempre durante la consulta, a todas las adolescentes sexualmente activas y principalmente a aquéllas que nunca fueron sometidas a este procedimiento, pero que ya empezaron su vida sexual. Debido a las altas tasas de atipias cervicales en este grupo de edad, la mayoría de los entrevistados ha coincidido en que la medida más eficaz para disminuir los índices de cáncer cervical en Brasil sería la mejora en la calidad de la consulta ginecológica, con el fin de implantar una rutina ginecológica desde la edad precoz.

En esta investigación se ha constatado que el mayor obstáculo a la consulta de las adolescentes es la carencia de una atención más detenida. El ideal sería que este tipo de atención pudiera realizarse por un profesional que se propusiera escuchar, acoger y acompañar a la joven (22). Es necesario, por lo tanto, ampliar el campo de percepción clínica, con el fin de buscar en otras áreas del conocimiento humano y científico, respuestas más adecuadas a las quejas y síntomas, bajo el riesgo de desarrollar una actuación superficial, ineficaz y de baja acción resolutiva (23). En la consulta, la buena relación con la adolescente es fundamental. Es siempre circundado de expectativa por la joven el profesional que la atenderá, en especial cuando se trata del ginecólogo (24).

Si consideramos las particularidades y las dificultades de la atención a este público, el entrenamiento de los profesionales tendría un impacto positivo en salud del adolescente. Éstos necesitan estar capacitados para informar, orientar y aconsejar a las adolescentes en cuestiones relacionadas a la sexualidad. Sería interesante una capacitación que se extendiera a todos los ginecólogos, ya que la mayoría de los servicios no dispone de funcionarios para la atención exclusiva de esta población.
En esta investigación se ha considerado esencial motivar al propio médico. La motivación y la dedicación en relación a sus actividades, muchas veces se encuentran comprometidas por el múltiple empleo provocado por los bajos sueldos (25).

En la consulta ginecológica es fundamental un profesional que posea empatía y que sea amable, de modo que pueda crear un ambiente que facilite la estimulación de la adolescente a asumir, poco a poco, la responsabilidad por su propia salud. Los ginecólogos deben prepararse para respetar la autonomía de libre elección y ofrecer información y acompañamiento adecuado para garantizar asistencia de calidad a la joven.

\section{CONCLUSIÓN}

Con el fin de brindar atención ginecológica de calidad para los adolescentes, se requiere que los médicos tengan una formación complementaria sobre las características específicas de este grupo de edad. El estudio también señala la importancia de instituir el examen colpocitológico como rutina para las mujeres a partir de la adolescencia, una vez que ocurre la iniciación sexual.

La consulta ginecológica de adolescentes se muestra como un espacio privilegiado en el que ellas encuentran no sólo la resolución de dolencias y patologías, pero también comprensión y apertura para el diálogo. Posibilita al profesional una gran oportunidad para brindar la orientación sobre cómo cuidarse, la adquisición de hábitos saludables, actitudes de protección de la salud, de prevención del embarazo no deseado y de las ETS. Se recomienda que la atención considere aspectos globales de la vida de la adolescente, para brindar una atención diferenciada.

\section{BIBLIOGRAFÍA}

1. Altmann $\mathrm{H}$. A sexualidade adolescente como foco de investimento político-social. Ed Revista (Belo Horizonte) 2007;46: 287-310.

2. Pereira SM. Contracepção hormonal oral na adolescência: uma boa opção [dissertação]. Rio de Janeiro: Faculdade de Ciências Médicas, Universidade do Estado do Rio de Janeiro; 2006.

3. Souza MM, Borges IK, Medeiros M, Teles SA, Munari DB. A abordagem de adolescentes em grupos: o contexto da educação em saúde e prevenção de DST. J Bras Doenças Sex Transm 2004;16:18-22.

4. Medronho R, Bloch KV, Luiz RR, Werneck GL (eds.). Epidemiologia. 2a ed. São Paulo: Atheneu; 2009.

5. Machado $\mathrm{MH}$. Os médicos no Brasil: um retrato da realidade. Rio de Janeiro: Fiocruz; 1997.

6. Vieira ALS, Amâncio Filho A, Machado MH, Moisés MNM, (orgs). Trabalhadores de saúde em números. 
Rio de Janeiro: Fiocruz; 1998.

7. Bittar OJNV. O mercado médico no Brasil. Rev Admin Púb 1999;33:55-66.

8. Renepontes P, Eisenstein E. Gravidez na adolescência: a história se repete. Adol \& Saúde 2005;2:11-5.

9. Martins LBM, Costa-Paiva L, Osisc MJD, Sousa MH, Pinto Neto AM e Tadini V. Conhecimento sobre métodos anticoncepcionais por estudantes adolescentes. Rev Saúde Pública 2006;40(1):57-64.

10. Taquette SR, Vilhena MM, Silva MM, Vale MP. Conflitos éticos no atendimento à saúde de adolescentes. Cad Saúde Pública 2005; 21:1717-25.

11. Pereira SM. Rompendo preconceitos sobre a utilização da anticoncepção de emergência para as adolescentes. Adol \& Saúde 2010;7:31-6.

12. Brasil. Ministério da Saúde. Anticoncepção de emergência: perguntas e respostas para profissionais de saúde. Brasília: Departamento de Ações Programáticas Estratégicas; 2005.

13. Organização Mundial de Saúde. Relatório mundial sobre violência e saúde: prevenção. Genebra: OMS; 2002.

14. Revzina NV, Diclemente RJ. Prevalence and incidence of human papillomavirus infection in women in the USA: a systematic review. Int J STD AIDS 2005;16 528-37.

15. Manhart LE, Holmes KK, Koutsky LA, Wood TR, Kenney $\mathrm{DL}$, Feng $\mathrm{Q}$, et al. Human papillomavirus infection among sexually active young women in the United States: implications for developing a vaccination strategy. Sex Transm Dis 2006;33:502-8.

16. Longatto Filho A, Etlinger D, Gomes NS, Cruz SV, Cavalieri MJ. Freqüência de esfregaços cérvico-vaginais em adolescentes e adultas: revisão de 308.630 casos.
Rev Inst Adolfo Lutz 2003;29:31-4.

17. Giles M, Garland S. A study of women's knowledge regarding human papillomavirus infection, cervical cancer and human papillomavirus vaccines. Aust N Z J Obstet Gynaecol 2006;46:311-5.

18. Society of Gynecologic Oncologists Education Resource Panel Writing group, Collins $\mathrm{Y}$, Einstein $\mathrm{MH}$, Gostout BS, Herzog TJ, Massad LS, Rader JS, Wright J. Cervical cancer prevention in the era of prophylactic vaccines: a preview for gynecologic oncologists. Gynecol Oncol 2006; 102: 552-562.

19. Gérvas J. La vacuna contra el virus del papiloma humano desde el punto de vista de la atención primaria en España. Rev Bras Epidemiol 2008;3:505-11.

20. American College of Obstetricians and Gynecologists. HPV Vaccine - ACOG Recommendations. Disponible en: http://www.acog.org/departments/dept_notice. cfm?recno=7\&bulletin=3945.

21. Moscicki A, Shiboski S, Hills N, Powell K, Jay N, Hanson $\mathrm{E}$, et al. Regression of low-grade squamous intraepithelial lesions in young women. Lancet 2004;364: 1678-83.

22. Diniz CS, Cunha CF, Ferreira RA. Medicina do adolescente: avaliação de uma experiência de ensino interdisciplinar. Rev Med Minas Gerais 2008;18:173-8.

23. Fernandes JCL. A quem interessa a relação médicopaciente? Cad Saúde Púb 1993;9:21-7.

24. Peremans L, Hermann I, Avonts D, Van Royen P, Denekens J. Contraceptive knowledge and expectations by adolescents: an explanation by focus groups. Patient Ed Counseling 2000;40:133-41.

25. Rodriguez-Neto E. Reorientando o setor saúde no Brasil: desafios para a prática e a educação médicas. Divulgação Saúde Deb 1996;14:66-74 